\title{
Carinal resection for adenoid cystic carcinoma of left main bronchus under cardio-pulmonary by pass
}

\begin{abstract}
Carinal resection with reconstruction poses a real challenge to thoracic surgeons and has high mortality and morbidity rates. Thoracic malignancies involving carina are very complicated as per the treatment involved because of very complex surgical intervention and very tedious anesthetic technique. Therefore, before offering this complex treatment modality, one must carefully consider the underlying pathology and chance of long-term survival. Good long-term results in N0 or N1 diseases, as in our study and in previous studies, confirm the necessity of performing surgery in selected patients with carinal involvement. ${ }^{1} \mathrm{We}$ present a case of carinal resection with right tracheobronchial anastomosis for the adenoid cystic carcinoma of left main bronchus (Stage III, T4 N1 M0) in 27-years-old young male highlighting procedural complexities and their redressal during and after surgery. Patient tolerated surgery well and overall recovery was very smooth and uneventful.
\end{abstract}

Keywords: Tracheal resection; Carinal resection; Carina; Adenoid cystic carcinoma; Bronchial tumor; Cardio-pulmonary bypass
Volume 8 Issue 7 - 2017

\author{
Pankaj Srivastava, ' Arvind Kumar, ${ }^{2}$ Belal Bin \\ Asaf $^{2}$ \\ 'Om Surgical Center \& Maternity Home, India \\ ${ }^{2}$ Thoracic Surgery, Sir Ganga Ram Hospital, India
}

\begin{abstract}
Correspondence: Pankaj Srivastava, Laparoscopic, Thoracic, Thoracoscopic \&VATS Surgeon Om Surgical Center \& Maternity Home, SA 17/3, P-4, Sri Krishna Nagar, Paharia, Ghazipur Road,Varanasi, UP, India, Pin 221007, Tel +91-542 2586191,+919415226817, Email drpankajbns@gmail.com
\end{abstract}

Received: October 26, 2017 | Published: December II, 2017

\section{Introduction}

Tumors involving the carina represent a challenge due to the complexity of airway reconstruction and management. The most important factors affecting the long-term outcome are the completeness of resection and the absence of nodal involvement. In experienced centers resection of carina may be a safe approach in highly selected patients, with acceptable morbidity and mortality and good long-term survival.

Left carinal pneumonectomy is indicated for tumors that involve carina and the left main stem bronchus. Lung cancers considered for carinal pneumonectomy are at least $\mathrm{T} 3$ or $\mathrm{T} 4$ and select patients with these advanced cancers will benefit from resection and reconstruction. Primary airway neoplasms such as adenoid cystic carcinoma and carcinoid tumors, and a variety of unusual rare tumors, are occasional indications for carinal pneumonectomy, also rarely performed for benign or inflammatory strictures.

\section{Case presentation}

A 27-years-old reformed smoker, non-alcoholic male presented with the recurrent episodes of hemoptysis with cough for the last eight months. Cough was nonproductive and had no diurnal and/or seasonal variation. He had a prolonged history of low grade fever with left sided chest pain. He had a history of weight loss and occasional breathlessness also. There was no history of dysphagia, hoarseness of voice, bone pain and convulsions. There was no family history of lung or airway cancer. Patient had an average built with clubbing grade II but no palpable lymphadenopathy. Trachea was shifted to the left and there was no breath sounds on the left. Chest x-ray revealed mediastinal shift, wide opacity of left hemithorax-left lung collapse, compensatory emphysema of right lung and elevated left dome of diaphragm (Figure 1a). CT scan of chest revealed occlusion of the left main bronchus with collapse of the left lung and ipsilateral cardiomediastinal shift with compensatory hyperinflation of the right lung (Figures $1 \mathrm{~b} \& 1 \mathrm{c}$ ). Bronchoscopy revealed mitotic growth completely occluding the left main bronchus with some satellite nodules (Figures 2a \& 2b). Bronchoscopy guided biopsy concluded tumor as adenoid cystic carcinoma of left main bronchus.
Therefore, the patient was considered for surgery with the clinical diagnosis of Stage III (T4 N1 M0) adenoid-cystic carcinoma of left main bronchus with carinal deposits with left lung collapse. After thorough discussion with the anesthesia team, two staged operation- left pneumonectomy with lymph node clearance followed by carinal resection with right tracheobronchial anastomosis under cardiopulmonary bypass was decided. In stage I, patient was positioned right lateral and chest was opened up through left posterolateral thoracotomy incision in fifth intercostal space. Left lung was found completely collapsed, fibrotic and densely adherent to the chest wall. After meticulous adhesiolysis, left pneumonectomy was performed. Left main bronchus was divided with stapler distal to the tumor. Lymph node dissection was performed before pneumonectomy. Thoracotomy was closed in layers.

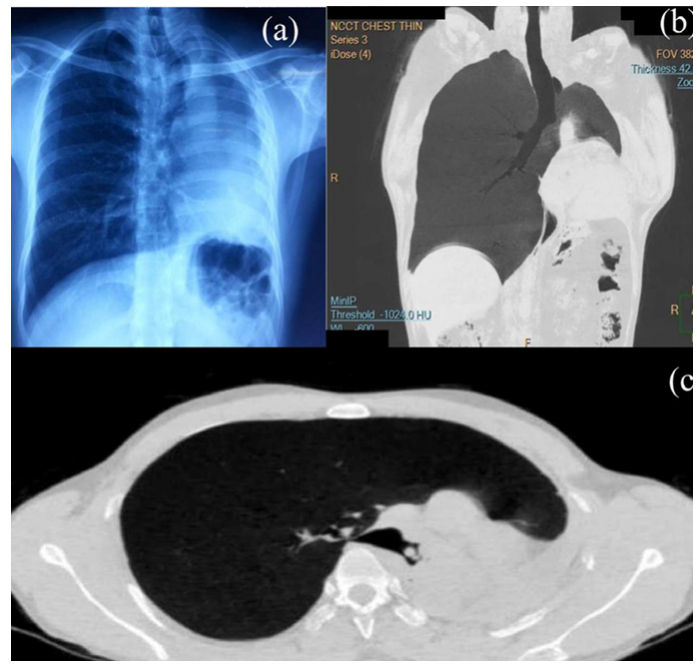

Figure Ia Chest x-ray posteroanterior view showing mediastinal shift, wide opacity of left hemithorax-left lung collapse, compensatory emphysema of right lung and elevated left dome of diaphragm.

Figure Ib \& c Non-contrast enhanced computer tomography (NCCT) of chest revealed occlusion of the left main bronchus with collapse of the left lung and ipsilateral cardiomediastinal shift with compensatory hyperinflation of the right lung. 


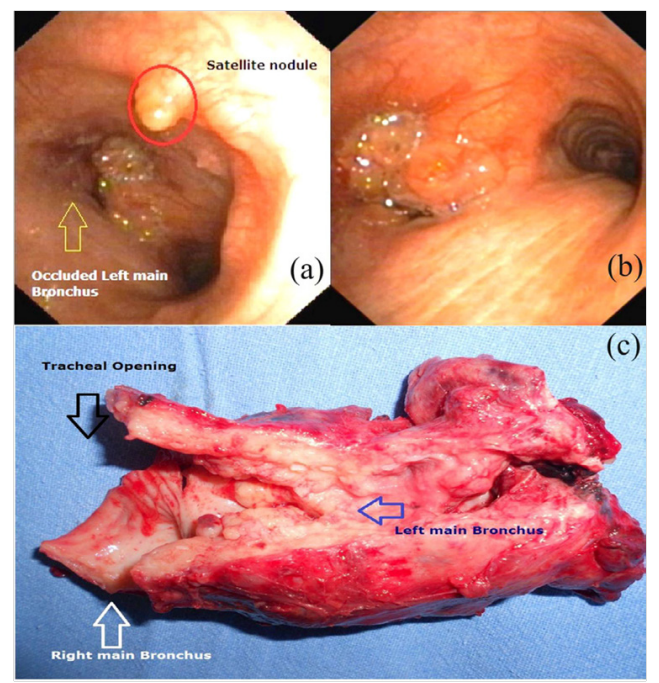

Figure 2a \& b Bronchoscopy revealed mitotic growth completely occluding the left main bronchus (yellow arrow) with some satellite nodules (red circle).

Figure 2c Resected specimen showing carina and tumor inside the left main bronchus with healthy margins of trachea and right main bronchus.

In stage II, patient was put in supine position. Median sternotomy was performed and lower trachea and carina were approached through the aortocaval space. After mobilizing the carina, right main bronchus and left main bronchus, cardiopulmonary bypass (CPB) between aorta and right atrium was established. All efforts were ardently taken to preserve the blood supply to the trachea and bronchus as much as possible. Trachea was divided $2 \mathrm{~cm}$ above carina and the right main bronchus was also divided $1 \mathrm{~cm}$ distal to carina. Therefore, the carina with $2 \mathrm{~cm}$ of the trachea, left main bronchus which was already stapled at stage I operation with tumor inside and $1 \mathrm{~cm}$ of the right main bronchus was excised (Figure 2c). All bronchial and tracheal resection margins were examined through frozen section. To mobilize the right main bronchus up, right inferior pulmonary ligament was divided and so as the pericardial attachments of pulmonary vessels by giving $U$-shaped incision of the pericardium underneath the hilum. End-to-end anastomosis with 3-0 polydiaxone was then established between right main bronchus and the trachea that was further covered with the vascularized flap of pericardial fat pad. Sternotomy wound was closed with $28 \mathrm{~F}$ drain which was removed on 5 th postoperative day. Two stay sutures were placed from chin to anterior chest wall (chin stitch), to keep the neck in flexed position postoperatively which were removed after 7 days. Jiang et al recommended two weeks stay for this guardian stitch. ${ }^{1}$. Patient was successfully extubated at the end of procedure. Postoperative period was uneventful except that he developed hoarseness of voice attributable to left recurrent laryngeal nerve injury. No specific measures were undertaken in post-operative care except, high protein diet, lung exercises and physiotherapy. Histopathologically, tumor was adenoid cystic carcinoma, grade I, main bronchus with nodal involvement (pT4N1pMx) whereas lung parenchyma show no cellular atypia and/or malignant cells. Patient was instructed to come for follow-up on every two weeks till 3 months and then monthly for next 3 months.

\section{Discussion}

Carinal resection with or without parenchymal resection, a rare surgical procedure, chiefly restricts its indication for neoplastic diseases in which the left carinal pneumonectomy further remains a very rare procedure with a mortality rate ranging from 2 to $16 \%{ }^{2}$
In 1963, Grillo et al. described resection of the distal trachea and carina to remove adenoid cystic carcinoma; the trachea was end-toend anastomosed to the right main bronchus, whereas the left main bronchus was anastomosed end-to-side to the trachea. ${ }^{3}$

Median sternotomy, currently the preferred approach for carinal resection, provides superb exposure to the tracheobronchial bifurcation, has less incisional discomfort, and results in less ventilatory restriction than a thoracotomy. ${ }^{4}$ The main disadvantages are that freeing pleuroparietal adhesions can be difficult, and mobilization of the left hilum requires cardiac retraction that might cause some hemodynamic instability., 4 In our case, pleuroparietal adhesions were expected, therefore, we decided to perform left pneumonectomy through left posterolateral thoracotomy first then carinal resection and tracheobronchial anastomosis through median sternotomy that also facilitated CPB. Recurrent laryngeal nerve (RLN) palsy, the known complication of the carinal resection estimated approximately $2.5 \%$ was also experienced in our case.

\section{Conclusion}

Although risky, when used with caution and enough surgical skill, carinal resection with reconstruction of airway can be the better option for increasing the disease-free-survival of patients with tumors involving carina with acceptable mortality and morbidity. Patients with malignant thoracic conditions involving carina should be offered surgical resection of lesion based on the extent of disease and the functional status. Positive N2/N3 lymph nodes may be considered as contraindication of the surgery because of poor prognosis and fatal outcome. ${ }^{6,7}$ Technical complexity of the operation warrants experience, expertise, multidisciplinary team work and good rescue infrastructure facility to achieve quality results.

\section{Conflicts of interest}

None.

\section{Acknowledgements}

None.

\section{References}

1. Jiang $\mathrm{F}, \mathrm{Xu} \mathrm{L}$, Yuan F, et al. Carinal Resection and Reconstruction in Surgical Treatment of Bronchogenic Carcinoma with Carinal Involvement. J Thorac Oncol. 2009;4(11):1375-1379.

2. Lorenzo Spaggiaria, Francesco Petrella, Domenico Galetta Carinal resection. MMCTS. 2012.

3. Grillo HC, Bendixen HH, Gephart T. Resection of the carina and lower trachea. Ann Surg. 1963;158:889-893.

4. Dutta R, Kumar A, Kaushal S, et al. A case of carinal schwannoma resected under cardiopulmonary bypass. Indian $J$ of Cancer. 2011;48(3):366-368.

5. Marc de Perrot, Elie Fadel, Olaf Mercier, et al. Long-term results after carinal resection for carcinoma: Does the benefit warrant the risk? $J$ Thorac Cardiovasc Surg. 2006;131(1):81-89.

6. Subasi M, Yazicioglu A, Gungor A, et al. A case of primary adenoid cystic carcinoma of trachea: a complex surgical approach with distal tracheal, carinal resection, right upper lobectomy and carinal reconstruction. Interactive CardioVascular and Thoracic Surgery. 2017;25(Suppl 1):ivx280.240.

7. Shin S, Park JS, Shim YM, et al. Carinal resection and reconstruction in thoracic malignancies. J Surg Oncol. 2014;110(3):239-44. 